\title{
Análisis del programa de formación de Cirugía Plástica y Reparadora de la Universidad de Chile: encuesta a egresados 2011-2017
}

\author{
Sergio Sepúlveda P. ${ }^{1}$, Diego Quispe V. ${ }^{1}$, Luis Selman B. ${ }^{2}$, \\ Enrique Larraín C. ${ }^{2}$, Stefan Danilla E. ${ }^{1}$, Cristian Erazo C. ${ }^{1}$, \\ Patricio Andrades C. ${ }^{1}$, Rolando Schulz R. ${ }^{1}$ y Claudia Albornoz G. ${ }^{1}$
}

\section{Analysis of the training program of Plastic and Reconstructive Surgery of the University of Chile: survey of graduates 2011-2017}

Background: The adequate plastic surgeons training demand a constant review and assessment of the training programs of residents. It has been 8 years since the first survey made to Plastic Surgery graduates of the University of Chile about the program training. Aim: Collect the opinion of Plastic Surgery graduates of the University of Chile between the years 2011-2017, in order to compare the results with previous generations. Materials and Method: A survey of 28 questions was conducted which evaluated general aspects of the residence, specific rotations of the resident, practical activities, theoretical activities and economic and research aspects. Results: There has been 21 graduates between 2011-2017, a survey response was obtained in 16 of them, $100 \%$ of the respondents approved the training program, $63 \%$ of respondents consider the duration of the program is insufficient. The best-evaluated rotation of the program was $\mathrm{HCUCH}$ according to $56 \%$ of graduates. The areas of best training perceived were body contour surgery and breast aesthetic surgery, respectively. Conclusion: Plastic surgery training program approval is still high in graduates; nevertheless the opinion about the needing for a more extensive program is kept. The analysis collected from this survey is an excellent tool to identify aspects of the training program can be improved.

Key words: plastic surgery; postgraduate; training program.

\section{Resumen}

Introducción: La formación de cirujanos plásticos de calidad exige una constante revisión y evaluación del programa aplicado. Han transcurrido 8 años desde que se evaluó la opinión de los egresados del Programa de Cirugía Plástica de la Universidad de Chile por primera vez. Objetivo: Recoger la opinión de los egresados del mismo programa entre los años 2011-2017, con el fin de cotejar los resultados con generaciones anteriores. Materiales y Método: Se realizó una encuesta de 28 preguntas, las cuales evalúan aspectos generales de la residencia, rotaciones específicas del residente, actividades prácticas, actividades teóricas y aspectos económicos y de investigación. Resultados: De un total de 21 egresados en el período comprendido 2011-2017 se obtuvo respuesta de la encuesta en 16 de ellos, el 100\% de los encuestados aprueba el programa de formación, el $63 \%$ de los encuestados considera que la duración del programa es insuficiente. La rotación mejor evaluada del programa fue el Hospital Clínico de la Universidad de Chile según un $56 \%$ de los egresados. Las áreas de mejor percepción de formación fueron cirugía de contorno corporal y cirugía estética mamaria respectivamente. Conclusión: La aprobación del programa de formación de la especialidad sigue siendo alta en los egresados, sin embargo, se mantiene la opinión de la necesidad de un programa más extenso. El análisis recogido de esta encuesta es una buena herramienta para identificar aspectos del programa que se pueden mejorar.

Palabras clave: cirugía plástica; postgrado; programa de formación.
'Departamento de Cirugía Hospital Clínico Universidad de Chile. Santiago, Chile. 2Internos Facultad de Medicina Universidad de Chile. Santiago, Chile.

Recibido el 26 de julio de 2018, aceptado para publicación el 29 de agosto de 2018.

Correspondencia a: Dr. Sergio Sepúlveda P. sepulveda.sep@gmail.com 


\section{Introducción}

Durante las últimas décadas hemos asistido a innovaciones del sistema de educación desde un sistema de docencia jerárquico vertical a un sistema más participativo, donde el alumno está involucrado en las distintas etapas o momentos del aprendizaje. En este nuevo escenario, se hace imprescindible poder recoger la opinión de los estudiantes involucrados respecto de su experiencia educativa.

En la actualidad, existen algunas herramientas objetivas, precisas y validadas para evaluar la calidad de un Programa de Postgrado. Uno de esos instrumentos es la realización de encuesta diseñada para indagar diferentes dominios, que nos permite analizar la percepción que tienen los egresados respecto del Programa realizado.

Han transcurrido 8 años desde que se evaluó la opinión de los egresados del Programa de Cirugía Plástica de la Universidad de Chile por primera vez ${ }^{1}$. Posterior al análisis de esos datos, se incorporaron algunas modificaciones y mejoras destinadas a optimizar las rotaciones de los residentes, creando también nuevas instancias docentes que se hace imperioso evaluar.

El objetivo de este artículo es recoger la opinión de los egresados del programa de Cirugía Plástica de la Universidad de Chile entre los años 20112017, de manera de poder analizar la calidad de este Programa de Formación, en forma integral y contrarrestar estos resultados, con aquellos obtenidos en encuestas realizadas a generaciones anteriores y publicadas en su momento, con esto podemos hacer una evaluación actualizada del Programa y del cuerpo docente involucrado.

\section{Materiales y Método}

Estudio descriptivo por medio de cuestionarios de tipo formulario Google el cual se diseñó con 28 preguntas las cuales evalúan aspectos generales de la residencia, rotaciones específicas del residente, actividades prácticas, actividades teóricas y aspectos económicos y de investigación.

Además, se evaluaron los distintos centros formadores incluidos en el programa en función de la percepción de los egresados. Se consultó sobre la duración de las rotaciones y cómo los egresados evalúan la utilidad de cada rotación en su formación. Además, se indagó respecto a la calidad e importancia de sus rotaciones en los diversos centros involucrados en el Programa de Especialidad derivada. Se consultó también por las actividades teóricas y prácticas efectuadas durante su formación.
El formulario Google fue solicitado a la totalidad de egresados del programa de formación en Cirugía Plástica, Reparadora y Estética de la Universidad de Chile entre los años 2011 y 2017.

La encuesta fue enviada por correo electrónico y por teléfono celular vía WhatsApp. Las respuestas fueron configuradas para garantizar el anonimato del encuestado y la no divulgación de opiniones personales. Los resultados fueron tabulados en planilla Microsoft Excel y los datos analizados con estadística básica.

\section{Resultados}

De un total de 21 egresados en el período comprendido 2011-2017 se obtuvo respuesta de la encuesta en 16 de ellos.

\section{Aspectos generales}

En general el Programa de Formación en Cirugía Plástica, Reparadora y Estética tuvo una evaluación satisfactoria (Figura 1).

El 69\% cree que durante la formación adquirió las destrezas suficientes para resolver las patologías que comprende la especialidad.

Con respecto a la duración actual de 2 años del programa de formación, un $63 \%$ piensa que es insuficiente y un $19 \%$ cree que es adecuado.

La mayoría cree que la duración total del programa de formación debiera ser de 3 años (Figura 2).

Con respecto a los objetivos del programa de formación, un $50 \%$ cree que no están claramente descritos y un $50 \%$ cree que el sistema de evaluación de conocimientos y destrezas que tiene el Programa no es el más adecuado.

Un $88 \%$ cree que los docentes estuvieron disponibles siempre que se les necesitó para aclarar dudas tanto teóricas como prácticas.

\section{Evaluación de rotaciones}

El 69\% de los encuestados cree que el número de rotaciones por distintos centros es el adecuado y un $25 \%$ desconoce cuál debería ser el número.

El 100\% de los egresados encontraron adecuada la infraestructura para la formación de los centros donde se realizan las rotaciones.

Con respecto a qué rotaciones piensan que deberían durar más, un $81 \%$ prolongaría la rotación en el Hospital Clínico Universidad de Chile (HCUCH) y un 56\% alargaría Quemados en el Hospital del Trabajador (Figura 3).

Con respecto a qué rotaciones debiesen acortarse, los encuestados estuvieron predominantemente en desacuerdo con acortar cualquier rotación. 


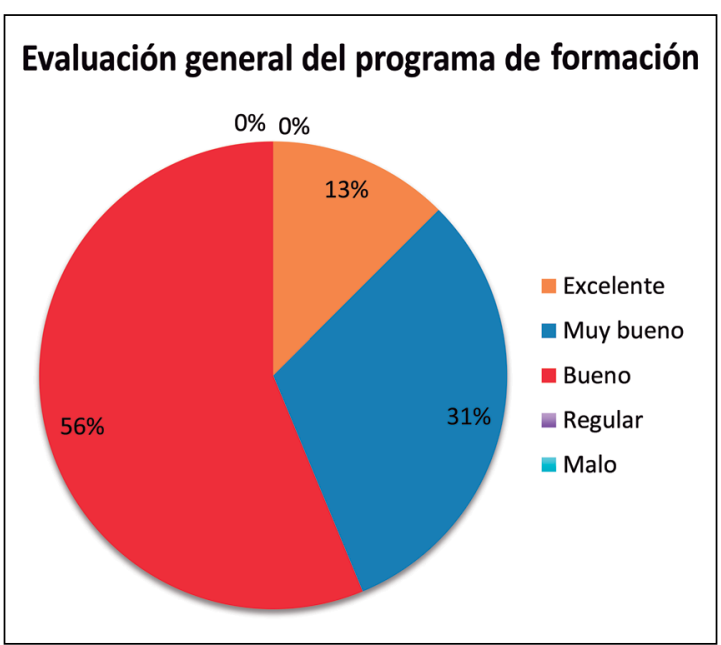

Figura 1. Análisis de la evaluación general que realizan los egresados del programa de formación. Pregunta realizada: ¿En general considero que el programa de formación en Cirugía Plástica, Reparadora y Estética es?

\section{Cuánto debería durar el programa de formación}

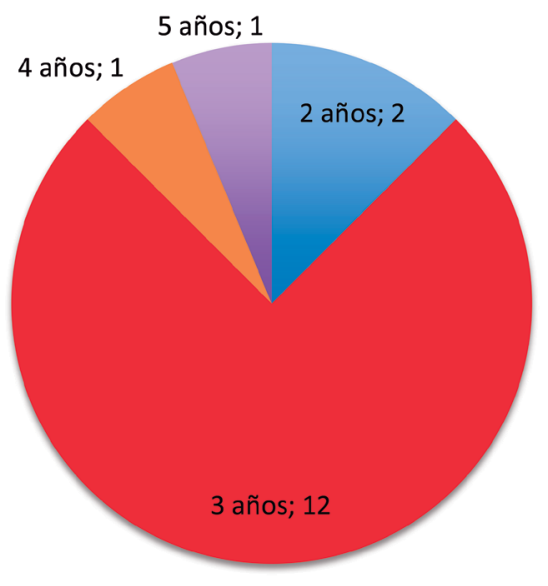

Figura 2. Cuánto debería durar el programa de formación según los encuestados. Pregunta realizada: Creo que la duración TOTAL del programa de formación en Cirugía Plástica, Reparadora y Estética debiera durar.

Las áreas mejor percibidas desde el punto de vista formación fueron cirugía de contorno corporal, cirugía estética mamaria y quemados. Las áreas que se percibieron con más falencias fueron cirugía maxilofacial y cirugía de mano (Figura 4).

Las rotaciones mejor evaluadas del programa fueron el Hospital Clínico, Quemados del Hospital del Trabajador y el Hospital Militar (Figura 5).

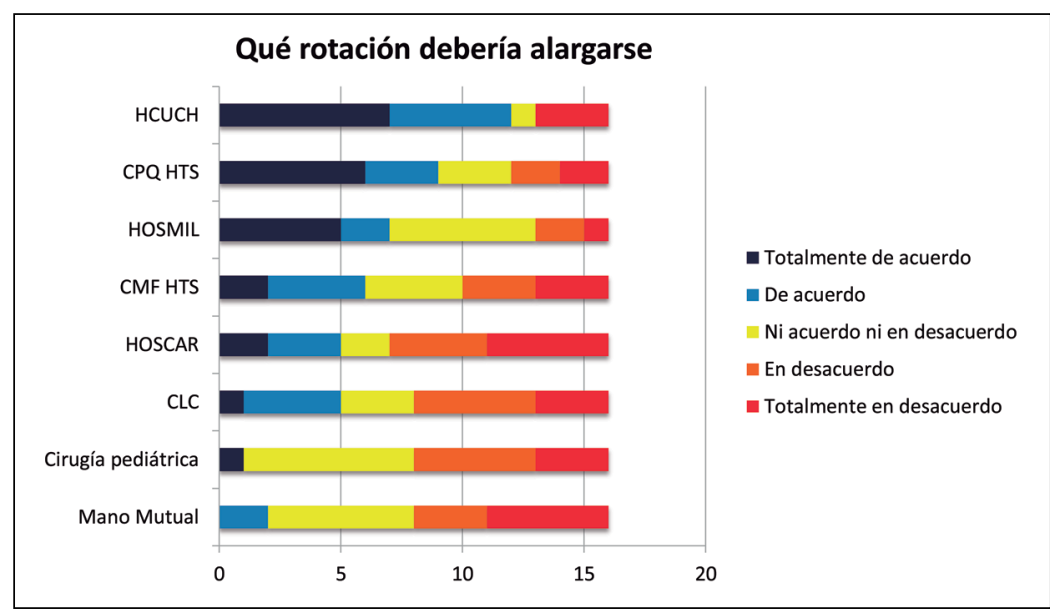

Figura 3. Análisis de las rotaciones que deberían alargarse según la opinión de egresados. Pregunta realizada: Creo que la(s) siguiente(s) rotaciones debiesen ALARGARSE. CPQ: Cirugía Plástica y Quemados, CMF: Cirugía maxilofacial, HOSMIL: Hospital Militar, HOSCAR: Hospital de Carabineros, CLC: Clínica Las Condes, HCUCH: Hospital Clínico de la Universidad de Chile. HTS: Hospital del Trabajador.

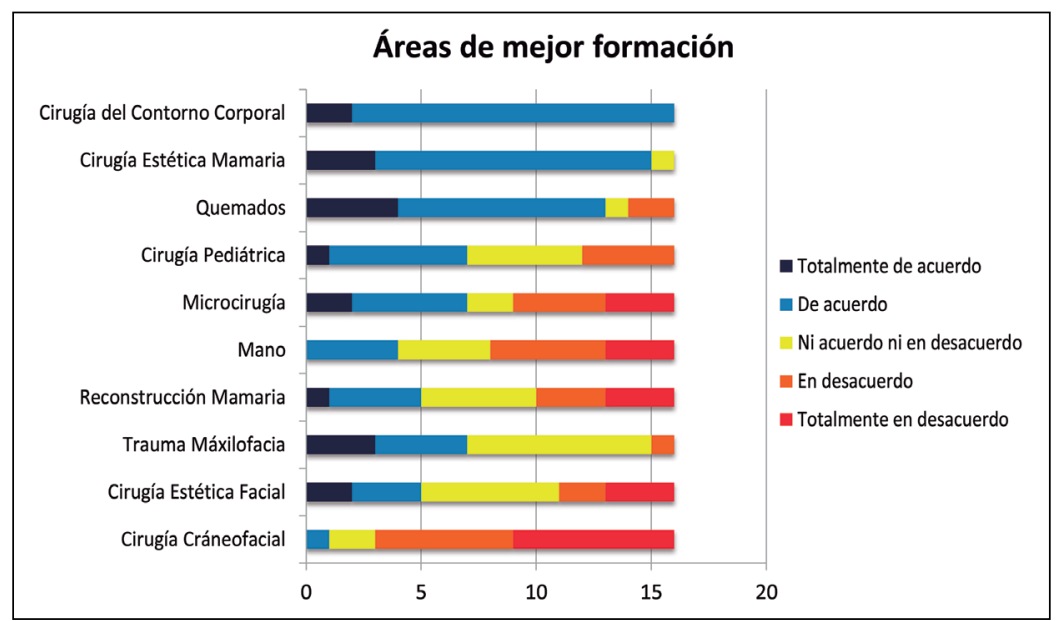

Figura 4. Conformidad respecto a la formación de distintas ramas de la subespecialidad. Preguntas realizada: Creo que la formación en las siguientes áreas fue la mejor que pude haber tenido.

\section{Actividades prácticas}

Al consultar cuántas participaciones en pabellón en los diferentes procedimientos quirúrgicos de la especialidad tuvo, $50 \%$ refiere entre $100-200,31 \%$ $200-300$ y $19 \%$ más de 500 . Un $63 \%$ consideró el número de participaciones en pabellón en los diferentes procedimientos quirúrgicos de la especialidad adecuado y un $38 \%$ insuficiente.

Con respecto a la variedad en cuanto a técnicas quirúrgicas en que tuvo oportunidad de participar, $56 \%$ lo encontró adecuado, 38\% insuficiente y 1 encuestado excesivo. 


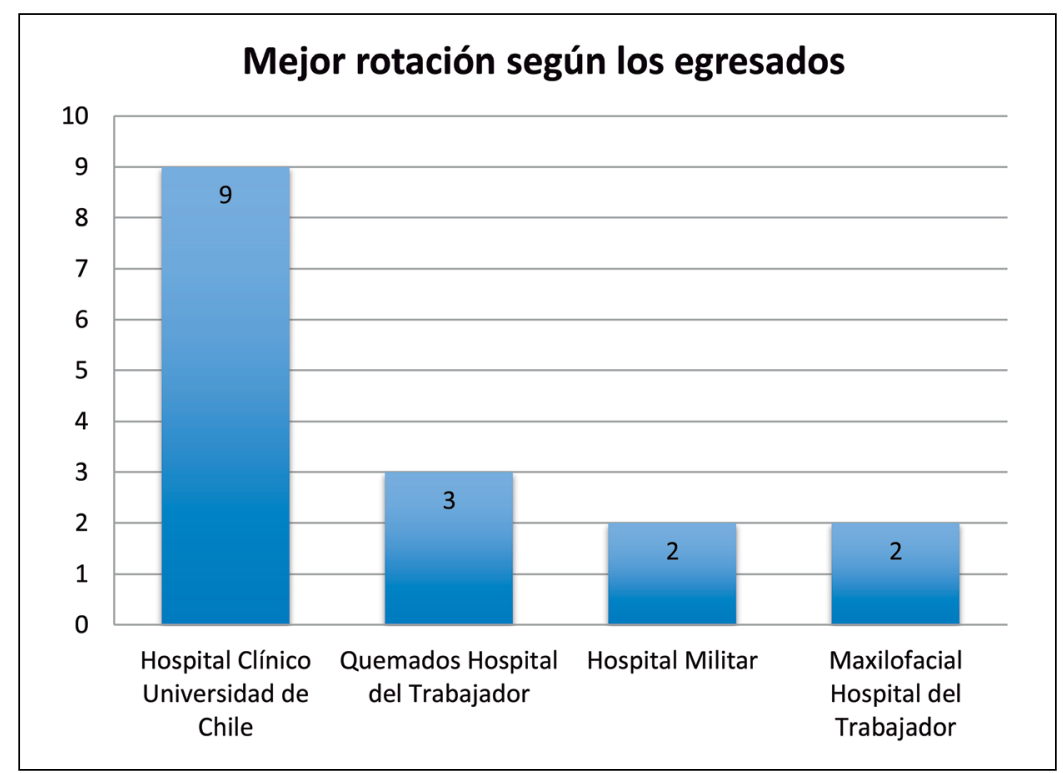

Figura 5. Rotaciones consideradas como la mejor del programa. Pregunta realizada: A su juicio cuál considera la mejor rotación del programa de formación en Cirugía Plástica, Reparadora y Estética.
$56 \%$ consideró importante participar en microcirugía experimental durante la formación y el otro $44 \%$ no está de acuerdo que sea necesario.

\section{Financiamiento}

Un 75\% cree que el cursar el Programa de Formación en Cirugía Plástica les genera dificultades económicas durante su realización. Con respecto a las fuentes más importantes de financiamiento durante el programa de formación, un $88 \%$ está muy de acuerdo o de acuerdo que fueron recursos personales y un $36 \%$ cree que ayudantías en cirugía plástica. Un $69 \%$ de los encuestados descartó el financiamiento otorgado por una institución.

Cuando se consultó cuántos años debería durar el programa de formación si éste fuera financiado, un $75 \%$ piensa que 3 años y $13 \% 2$ años.

\section{Discusión}

La evaluación constante de los programas de formación de todas las especialidades médicas es fundamental para mantener el ritmo de desarrollo de acuerdo a los nuevos conocimientos y tecnología. Esto se hace más relevante cuando los Programas de formación son colaborativos, es decir cuando la formación se realiza a través de diferentes rotaciones por diferentes hospitales. Lo anterior está en estricta relación con que no existe un solo centro que reúna el dominio de todas las áreas de la cirugía plástica, como son quemados, maxilofacial, cirugía plástica pediátrica, entre otros.

La opinión de egresados que se encuentran ejerciendo la especialidad, es un parámetro de enorme utilidad para evaluar la excelencia de la especialidad.

Un aspecto positivo de la encuesta realizada es que el $100 \%$ de los egresados considera el programa de formación como bueno o muy bueno, a diferencia de la opinión de los egresados entre el período 20042010 en que solo el $72 \%$ tenía esa percepción ${ }^{1}$. Otro aspecto importante a destacar es la duración del Programa de Formación, al igual que las encuestas efectuadas entre los egresados del período 2004-2010, la mayoría opina que la duración del Programa debería ser de 3 años. En 1992 el Consejo de Acreditación para la Educación Médica de Graduados (ACGME) creada en 1981 en USA, reconoce y acredita el formato de residencia de cirugía plástica integrado de 6 años, siendo el actual modelo de educación de la especialidad $^{2}$. Los defensores del programa de cirugía plástica integrado argumentan que un mayor tiempo de dedicación en el entrenamiento específico de ci- 
rugía plástica es necesario para abordar la amplitud de conocimiento, la complejidad de los pacientes y la experiencia quirúrgica requerida para la práctica competente $^{2}$. Aquellos que apoyan la formación tradicional, con un programa de entrenamiento de cirugía general previo, argumentan que una base en cirugía general le permite a los residentes tener una mayor exposición quirúrgica, reconocer con certeza la especialidad que quieren seguir y una mayor madurez perioperatoria cuando inician su entrenamiento en cirugía plástica ${ }^{3}$. Roostaeian y $\mathrm{col}^{3}$ realizaron una encuesta a 5.400 miembros de la Sociedad Americana de Cirugía Plástica concluyendo que los residentes de un programa integrado fueron vistos con mayor conocimiento, pero con menos habilidad quirúrgica y experiencia en investigación. La constante opinión de los egresados de Cirugía Plástica de la Universidad de Chile, habla de la necesidad de un programa de formación más extenso, donde se deberá revisar en profundidad el modelo de formación actual, que probablemente deba ser readecuado a un formato de mayor duración.

Algunos aspectos alarmantes de los datos obtenidos es que un 50\% cree que los objetivos del programa de formación no están claramente descritos y que el sistema de evaluación de conocimientos y destrezas que tiene el Programa actual, no es el más adecuado.

La determinación de competencias y logros de aprendizaje definidos, es un aspecto fundamental en los Programas de Formación modernos, pues ayudan a identificar el contenido del programa, estructurar el estudio y permiten guiar la selección de las actividades y evaluaciones más significativas y relevantes ${ }^{4}$. Conforme a los datos que se desprenden de esta encuesta, es necesario reforzar y difundir de un modo más elocuente y directo, la estructura específica del Programa de Estudio de Cirugía Plástica. El año 1999 el ACGME introduce 6 dominios para las competencias clínicas centrales de la profesión médica e introduce un sistema de evaluación que ya no es basado en el cumplimiento de las rotaciones sino basado en resultados demostrados ${ }^{5}$. El año 2009 la ACGME reestructura el sistema, estableciendo subdominios por especialidad, creando un nuevo sistema de evaluación, en el cual se evalúa la realización de hitos en la formación, los cuales debe cumplir el residente. Este sistema establece 5 niveles de manera que cuando el residente completa los hitos del nivel 1 pasa a cursar los del nivel 2 y así sucesivamente ${ }^{6}$. En julio de 2015 el ACGME publica los hitos que hay que cumplir en el Programa de Formación de Cirugía Plástica ${ }^{7}$.

Determinar en forma concreta las competencias que debe adquirir un residente en los distintos ámbitos de la especialidad facilita la planificación de métodos de evaluación transparentes y reproducibles. Existen variados métodos de evaluación descritos, tales como: formularios de calificación global, evaluación en $360^{\circ}$, exámenes orales con casos que al residente le tocó enfrentar o casos ficticios, examen de preguntas de opción múltiple, lista de verificación de competencias adquiridas, encuestas de pacientes, portafolio de logros, modelos y simulaciones, examen OSCE y confecciones de rúbricas, entre los métodos más utilizados ${ }^{5,8}$.

Otro punto valorado por los egresados, es la participación en microcirugía experimental durante la formación. Las simulaciones y los modelos se emplean cada vez más en diferentes ámbitos en la formación de postgrado. Los residentes de cirugía plástica de los últimos años han podido utilizar modelos sintéticos o modelos animales de experimentación en diferentes cursos de microcirugía y unidades de simulación.

La constante opinión de los egresados en las encuestas realizadas durante el período 2004-2010 ${ }^{1}$ y 2011-2017 invita a replantear los métodos de evaluación que se utilizan actualmente y postular métodos más modernos que permiten una evaluación más objetiva y transversal, pero para ello es fundamental establecer objetivos específicos de aprendizaje en cada rotación.

Con respecto a la evaluación de las rotaciones, un $81 \%$ está de acuerdo y muy de acuerdo en prolongar la rotación en el Hospital Clínico Universidad de Chile y un 56\% alargaría la rotación de Quemados en el Hospital del Trabajador.

Cuando se les consultó en qué áreas recibió la mejor formación que pudo haber tenido, las áreas que presentaron un mayor porcentaje de desacuerdo fueron cirugía craneofacial y cirugía de mano, exactamente las mismas rotaciones que en la encuesta realizada por Benitez y col${ }^{1}$, lo que por una parte indica que se mantiene la calidad de enseñanza en algunos centros, pero plantea también la necesidad urgente, de revisar los contenidos, objetivos, instancias académicas y de evaluación de las rotaciones donde los encuestados cree que se podría mejorar.

La mayoría de los egresados refiere haber tenido un número de participaciones en procedimientos quirúrgicos entre 100 y 300 , con un porcentaje como primer cirujano muy variable entre los encuestados, sin embargo, esta encuesta al ser retrospectiva está muy propensa a presentar sesgo de memoria. Existen escasos estudios que reportan el número de cirugías realizadas por los residentes de cirugía plástica, un estudio realizado en Reino Unido ${ }^{9}$ que recopila 
los procedimientos quirúrgicos realizados por los residentes, registrados en forma obligatoria por medio de un programa de dispositivo móvil llamado elogbook, refiere que en 6 años del programa de formación entre los años 2010 y 2014, los residentes participaron en promedio en 2.117 procedimientos, de los cuales realizaron como primer cirujano un promedio 1.571 procedimientos.

El aprendizaje de habilidades quirúrgicas encarna grandes desafíos en la educación de residentes contemporáneos. La presión de los campos clínicos de generar ingresos sustentables, el énfasis en el uso económico del pabellón y las preocupaciones normativas y medico legales afectan negativamente en las oportunidades quirúrgicas del residente ${ }^{10}$. Koulaxouzidis y col$^{11}$ evalúan la seguridad y satisfacción de los pacientes en 273 procedimientos estéticos realizados por residentes de cirugía plástica, concluyendo que las cirugías estéticas realizadas por residentes de cirugía plástica bajo la supervisión de sus profesores es segura y proporciona altos niveles de satisfacción del paciente en el postoperatorio. Sasor SE y col ${ }^{12}$ estudian el costo quirúrgico de cirugías de labio leporino y paladar hendido cuando estos son efectuados por un residente $v s$ un staff del equipo, identificando que cuando fueron realizadas por residentes hubo un $60 \%$ más tiempo operatorio en labio leporino y un $65 \%$ más tiempo operatorio en paladar hendido. Al realizar la conversión monetaria se calculó un costo de oportunidad de más de \$ 275 dólares por caso efectuado por un residente. El Programa de Cirugía Plástica de la Universidad de Chile fue constituido considerando la fundamental importancia que tiene la práctica quirúrgica de los residentes, si bien esto presenta un costo económico no menor, es un sacrificio que la Universidad y los campos clínicos están dispuestos a asumir en aras de la formación de cirujanos plásticos competentes a la necesidad del país. Sin bien existe un costo económico debido a la ejecución de procedimientos quirúrgicos realizados por residentes, estos se ponderan frente a los beneficios que entrega el contar con residentes desde el punto de vista de control estricto y constante de los pacientes, investigación clínica y el estímulo de excelencia u orgullo que entrega a los staff que se desempeñan como profesores. La variabilidad de opinión de los egresados frente a las cirugías realizadas y la necesidad de una monitorización más cercana de los procedimientos quirúrgicos efectuados por residentes invita a idear un método de registro más eficiente. Una aplicación telefónica que registre los procedimientos realizados por el residente en una base de datos podría ser una alternativa fácil de implementar, que permitiría entregar información muy útil a la hora de evaluar si los residentes están realizando un número adecuado de cirugías en los distintos ámbitos de la especialidad.

Cuando se consultó sobre la variedad en cuanto a técnicas quirúrgicas en que tuvo oportunidad de participar el egresado, un número significativo lo describe como insuficiente, una alternativa a esta deficiencia es la aplicación del videoforo como estrategia didáctica de discusión de técnicas quirúrgicas. Los videos grabados se pueden usar para análisis de los pasos de la cirugía, revisión de la técnica empleada y evaluación del uso del instrumental ${ }^{13}$.

Las actividades académicas teóricas del programa presentaron un gran avance con la implementación del curso periódico "Cirugía Plástica Esencial”, el $100 \%$ de los encuestados estuvo muy de acuerdo o de acuerdo que es necesario como actividad teórica en el programa de formación de la especialidad. Este gran porcentaje de aprobación propone la creación de instancias académicas similares que aborden otros temas específicos de la especialidad.

Una nueva instancia docente, al igual que el curso Cirugía Plástica Esencial, que permite al residente participar de una jornada educativa de alto nivel, con un planteamiento didáctico, ha sido la reunión mensual en horario extraacadémico denominada como "Journal Club", donde residentes y académicos expertos, analizan y discuten artículos médicos seleccionados.

Un número significativo de egresados encuentra importante realizar investigación clínica durante la residencia de Cirugía Plástica, sin embargo, cuando se preguntó si tuvieron las oportunidades y facilidades para realizar investigación clínica el 50\% estuvo en desacuerdo. Considerando la importancia de la medicina basada en la evidencia en la actualidad, se hace imperioso buscar caminos para fomentar y facilitar la investigación clínica de los residentes en los centros asistenciales y docentes. El año 2015 se implementa en el Departamento de Cirugía del Hospital Clínico de la Universidad de Chile, la creación de un "Fellow de Investigación en Cirugía Plástica", el cual tiene el potencial de colaborar activamente para facilitar la investigación clínica de los residentes. Fomentar un nexo más fluido y constante entre el fellow de investigación y el residente podría ser una buena alternativa para que los residentes que presenten una pregunta clínica puedan elaborar y llevar a cabo un diseño de investigación.

Con respecto al financiamiento llama la atención que la mayoría de los egresados financiaron su período de formación con recursos personales descartando el financiamiento de parte de una institución, realidad que ha experimentado una importante 
transformación desde que se implementó el plan de ingreso, formación y retención de especialistas en el sector público ${ }^{14}$.

\section{Responsabilidades éticas}

Protección de personas y animales. Los autores declaran que para esta investigación no se han realizado experimentos en seres humanos ni en animales.

Confidencialidad de los datos. Los autores declaran que en este artículo no aparecen datos de pacientes.

Conflictos de interés: no hay.

\section{Referencias}

1. Benítez S, Andrades P, Danilla S, Erazo C, Sepúlveda S, Figueroa M. Evaluación de Programa de Especialidad de Cirugía Plástica, Reparadora y Estética de la Universidad de Chile. Visión de los últimos 6 años por egresados. Rev Chil Cir. 64:523-7.

2. Schneider LF, Barr J, Saadeh PB. A Nationwide Curriculum Analysis of Integrated Plastic Surgery Training: Is Training Standardized? Plast Reconstr Surg [Internet]. 1 de diciembre de 2013 [citado el 18 de mayo de 2018];132(6). Disponible en: https://insights.ovid.com/ pubmed?pmid $=24281610$.

3. Roostaeian J, Fan KL, Sorice S, Tabit CJ, Liao E, Rahgozar P, et al. Evaluation of Plastic Surgery Training Programs: Integrated/combined versus Independent. Plast Reconstr Surg [Internet]. 1 de julio de 2012 [citado el 18 de mayo de 2018];130(1). Disponible en: https://insights.ovid.com/ pubmed?pmid=22743900.

4. Northern Illinois University-Faculty
Development and Instructional Design Center. writing goals and objectives [Internet]. Disponible en: www.niu.edu/ facdev.

5. Bancroft GN, Basu CB, Leong M, Mateo C, Hollier LH, Stal S. Outcome-Based Residency Education: Teaching and Evaluating the Core Competencies in Plastic Surgery: Plast Reconstr Surg. 2008;121:441e-448e.

6. Sillah NM, Ibrahim AMS, Lau FH, Shah J, Medin C, Lee BT, et al. The New Accreditation Council for Graduate Medical Education Next Accreditation System Milestones Evaluation System: What Is Expected and How Are Plastic Surgery Residency Programs Preparing? Plast Reconstr Surg. 2015;136:181-7.

7. The Plastic Surgery Milestone Project. J Grad Med Educ. marzo de 2014;6(1s1):225-42.

8. Davis D, Lee G. The Use of Standardized Patients in the Plastic Surgery Residency Curriculum: Teaching Core Competencies with Objective Structured Clinical Examinations: Plast Reconstr Surg. 2011;128:291-8.
9. Highton L, Lamb A, Fitzgerald A, Wood $\mathrm{S}$, Lees V, Winterton R. An analysis of the operative experience of plastic surgery trainees in the United Kingdom using eLogbook. J Plast Reconstr Aesthet Surg. 2017;70:1464-71.

10. Luce EA. The Future of Plastic Surgery Resident Education: Plast Reconstr Surg. marzo de 2016;137:1063-70.

11. Koulaxouzidis G, Momeni A, Simunovic F, Lampert F, Bannasch H, Stark GB. Aesthetic Surgery Performed by Plastic Surgery Residents: An Analysis of Safety and Patient Satisfaction. Ann Plast Surg. 2014;73:696-700.

12. Sasor SE, Flores RL, Wooden WA, Tholpady S. The Cost of Intraoperative Plastic Surgery Education. J Surg Educ. 2013;70:655-9.

13. Loukas C. Video content analysis of surgical procedures. Surg Endosc. 2018;32:553-68.

14. Plan de ingreso, formación y retención de especialistas en el sector público de salud [Internet]. 2015. Disponible en: http:// www.minsal.cl/ medicosespecialistas/ 